\title{
Morphologie traditionnelle de la société albanaise
}

L'organisation traditionnelle de la société qui, jusqu'aux années 1930, faisait partie des modes de vie du paysage albanais, inspirant bon nombre de récits et de relations de voyage, ne correspond plus à la réalité sociologique de l'Albanie contemporaine. La littérature, le cinéma, une 'folklorisation' au service de multiples causes identitaires se chargent désormais d'entretenir avec ferveur toute une imagerie des racines ethniques et culturelles de la Nation. Les représentations deviennent elles-mêmes objets de culte. Durant les dernières décennies, l'imposition du régime communiste suivie de l'intrusion récente de la société industrielle et de l'économie de marché dans le monde rural albanais ont parachevé ce à quoi plusieurs siècles de volonté politique ottomane n'avaient pu parvenir.

On se propose ici d'éclairer l'organisation sociale traditionnelle qui est longtemps demeurée constituée de groupes élémentaires plutôt fermés, c'est-à-dire orientés de façon nécessaire par la naissance des individus. L'unité de base était la shpi ou shtëpi 'maison', nommée aussi zjarr 'feu', vatër 'foyer', tym 'fumée', oxhak 'cheminée' (cf. angl. household, fr. maisnie, gr. nikokireta, roum. gospodãrie, turc. hane), à laquelle on attribue la valeur moyenne de cinq individus et que l'on peut comparer au 'feu' du Moyen Âge occidental. De règle générale, les occupants d'une shtëpi, 'maison', sont reliés par des rapports de parenté et ils ont une propriété et une vie commune. Cela confère une stabilité interne à la famille et fait d'elle une forme immuable de l'organisation sociale.

\section{Le modèle segmentaire et ses limites}

Surtout dans les régions où les droits coutumiers étaient particulièrement respectés, comme dans M alësia e M adhe, les Grandes Montagnes du Nord, dans les Montagnes de Labëria au Sud-Ouest, mais aussi dans les campagnes de Myzeqe à l'Ouest, jusque assez récemment, dans les grandes shpi, shtëpi, vivaient des familles d'un type qu'on peut appeler, à la suite de Claude Lévi-Strauss, plutôt domestique que conjugal (1983: 77). Elles reposaient sur des mécanismes complexes et rigides d'organisation. Elles étaient caractérisées comme un groupe social à fonctions multiples: économique, politique, juridique et coutumier.

Dans ce type de familles la coopération familiale et parentale est presque entièrement fermée sur elle-même. Les enfants aident leurs parents et deviennent responsables des travaux quotidiens dont ils assument la charge. Ce faisant ils s'identifient peu à peu à leurs futurs rôles. L'organisation familiale repose, encore récemment, sur la cohabitation du père et des fils mariés, les fils ne se séparant qu'après la mort du père. Les rapports intra-familiaux sont marqués par une double relation de 
domination: domination des hommes sur les femmes et domination des vieux sur les jeunes. L'autorité du chef de famille est totale, bien que son pouvoir ne soit pas véritablement absolu, en ce sens que la propriété est collectivement celle du groupe et qu'il ne peut en aucune manière en disposer ou l'aliéner à sa guise. En tout cas, un fils ne contredira jamais son père et, parmi les Albanais de Cossovie, aujourd'hui encore, jamais un fils, même ayant suivi les études les plus poussées, ne quittera le groupe familial contre la volonté paternelle, sauf à accepter rupture et rejet, ce qui se produit rarement. Les formes du respect sont complexes et très strictement observées.

En revanche, dans la société traditionnelle des Slaves du Sud, le modèle dominant est celui d'une communauté familiale basée sur la cohabitation des frères, même en dehors de la présence vivante du père. Ce type d'organisation, connu sous le nom de zadruga, a donné lieu a une abondante littérature ethnologique, où il a été souvent présenté comme un modèle de démocratie familiale égalitaire, au moins entre les hommes (Sicard 1943). La maison, c'est-à-dire le groupe domestique, la communauté de vie et l'exploitation agricole, apparait toujours comme l'élément central de cette organisation sociale, à travers les comportements et à travers les manifestations symboliques. Mais si la société slave est organisée selon un principe horizontal: importance des relations collatérales, primauté du tangible et du contemporain, dans la société albanaise domine le principe vertical qui soumet le bas, le jeune, le présent, le fils, au haut, à l'ancien, au passé, au père.

Dans cette société traditionnelle, l'individu se définit par son appartenance à différentes relations sociales emboîtées les unes dans les autres. L'identité se rapporte à plusieurs niveaux qui mettent souvent en contradiction principe de parenté et principe de résidence. L'appartenance de type tribal ou lignager est parfois primordiale et transcende le cadre villageois ou territorial. La structure sociale est souvent fondée sur une partition spatiale du territoire, habité par des groupes lignagers avec référence tribale.

Les vestiges d'un type d'organisation lignagère sont notés avant tout dans certaines régions isolées au Nord de l'Albanie. Les récits et les études concernant ces régions sont plus nombreux et plus accessibles. Les descriptions de voyageurs (Hahn 1854; Hecquard 1858; Degrand 1901; Baldacci 1917) ou de missionnaires (Gjeçov 1989; Cozzi 1910, 1944; Valentini 1956, 1965), aussi bien que les recherches d'anthropologues (Durham 1909, 1928; Hasluck 1954; Nopcsa 1925; Coon 1950) ou de démographes (Seiner 1922), sont complétés par des études récentes historiques (Pulaha 1975, 1977), ethnographiques (Zojzi 1977; Ulqini 1977, 1985, 1988) et juridiques (Pupovci 1971; Elezi 1983). Des circonstances géographiques et historiques ont effectivement permis à ce type d'organisation de se maintenir plus longtemps dans le Nord. La population, connue le plus souvent sous l'appellation guègue, recluse dans ses montagnes, était plus attachée au système lignager que la population dite tosque au Sud du fleuve Shkumbini, qui vivait dans un espace beaucoup plus ouvert, où les chaînes de montagne étaient séparées par de larges bassins fertiles, facilitant les communications interrégionales et le développement urbain. Cette ouverture vers l'extérieur, accompagnée des effets d'une tradition migratoire très développée et des contacts avec les voisins grecs, eut une influence sur les modes de vie et provoqua un relâchement de l'observance des lois coutumières. Néanmoins, l'équivalent approximatif du même type d'organisation sociale a pu être attesté dans une partie des régions du Sud-Ouest aussi, plus précisément dans les Montagnes de Labëria (Ulqini 1987; Elezi 1994).

Mais si les auteurs contemporains se livrent à une analyse critique des sources, dans 
le meilleurs des cas, ils ne font qu'une nouvelle compilation anthologique des mêmes données, qui certes peut livrer des aperçus précieux et corriger les théories scientifiques avancées par les savants officiels. Qu'ils se considèrent comme spécialistes de la région (Stahl 1988) ou qu'ils soient titulaires de chaires universitaires spécialisées (Daniel 1989), ils n'arrivent pas toujours à renouveler l'analyse pour essayer de dégager dans le détail l'essence structurelle de ce type d'organisation sociale.

L'unité sociale principale est appelé fis(i) au Nord, qui est un emprunt relativement récent au grec moderne $\phi v \sigma \iota \sigma$ 'nature, caractère', développé tardivement en terme juridique et social de parenté à l'intérieur de l'albanais (Çabej 1996: 194). À ce terme largement utilisé dans la littérature ethnologique, malgré sa restriction régionale et temporelle, répond fara, littéralement 'grain, semence', développé en terme de parenté pour exprimer une entité sociale de type parental ou lignager. Le terme est plus spécialement utilisé au Sud-Ouest de l'Albanie, mais il est connu dans toute l'aire de peuplement albanais et dans la plupart des autres langues sud-est européennes (Çabej 1996: 142-5). Le terme générique même de la 'parenté' en albanais farefisi est un néologisme composé à partir des deux termes plus anciens fara et fisi. En tout cas, l'unité sociale appelée en particulier fisi au Nord apparaît comme un groupe de filiation, dont les liens avec l'ancêtre éponyme sont fictifs ou imprécis, mais globalement admis. Chacune de ces unités a une origine, connue ou reconnue, souvent datée en nombre d'années ou de générations, et une histoire: celle de l'établissement du fondateur dans la région qu'occupe le fisi, et des divisions ou des fusions avec d'autres groupes de même nature.

Un territoire donné, défini par des limites géographiques ou politiques, n’est ainsi jamais occupé par un seul groupe de descendance, mais par une pluralité de groupes fondés sur la parenté, qui se répartissent en trois catégories. Les pushtues, ou 'conquérants', constituent le groupe dominant. Ils constituent un lignage (fisi), qui a fini par s'identifier au territoire. Les anas, littéralement ceux 'd'à côté', sont ceux que les 'conquérants' trouvent à leur arrivée et dont les descendants sont encore nettement distingués. Les të ardhur, ou 'nouveaux venus', sont les gens arrivés une fois les 'conquérants' établis et reconnus comme dominants. Une communauté de nouveaux venus forme aussi un groupe de descendance, rarement un lignage (fisi). Son fondateur est membre d'une section de lignage ayant quitté son territoire d'origine. Il s'installe sur le territoire d'un lignage dominant à condition qu'il en respecte les lois. Ses descendants, comme les anas, seront à la fois reconnus et ditingués.

Des communautés géographiquement distantes se réclament fréquemment d'un même ancêtre, et font ainsi partie d'un même lignage. Parfois plusieurs lignages, tous dominants dans leurs territoires respectifs, se reconnaissent un ancêtre commun. Chacun d'eux descend d'un des fils de cet ancêtre. Les descendants de ces fils forment les këmbë, littéralement les 'jambes, piliers', du lignage. Lorsque tous les fils sont restés sur le même territoire, celui-ci est divisé en autant de parts qu'il y a de fils. Chaque këmbë entretient avec son territoire les mêmes rapports que le fisi avec le sien. Groupe de descendance et groupe territorial ne correspondent pas exactement, mais le groupe territorial porte le nom du groupe de descendance le plus important. Lorsque, au contraire, un ou plusieurs fils du fondateur quittent le territoire du père et partent s'établir ailleurs, leurs descendants peuvent ou bien constituer un këmbë du lignage d'origine, s'ils deviennent des të ardhur, 'nouveaux venus', dans le territoire d'un autre lignage, ou bien former un nouveau fisi, s'ils deviennent des pushtues 'conquérants' dans un territoire qui portera leur nom. 
Chaque këmbë porte un nom, souvent celui de son fondateur, et un numéro. Le këmbë issu du fils aîné du fondateur du lignage sera le premier këmbë (këmbë e parë), celui du deuxième fils, le deuxième këmbë (këmbë e dytë), et ainsi de suite. Ce numéro détermine le rang du këmbë dans les assemblées communes. Le këmbë, qui s’appelle parfois vllazni e madhe, 'grande phratrie', ou çetë, 'détachement, unité', peut se diviser à son tour en vllazni e vogël, 'petite phratrie'. Mais cette subdivision ne se rencontre pas partout et ne semble pas jouer un rôle important. Ce n'est pas une unité pertinente dans les assemblées et elle n'a pas de correspondant territorial qui porte son nom.

La troisième unité d'identification importante, après le fisi et le këmbë, est la

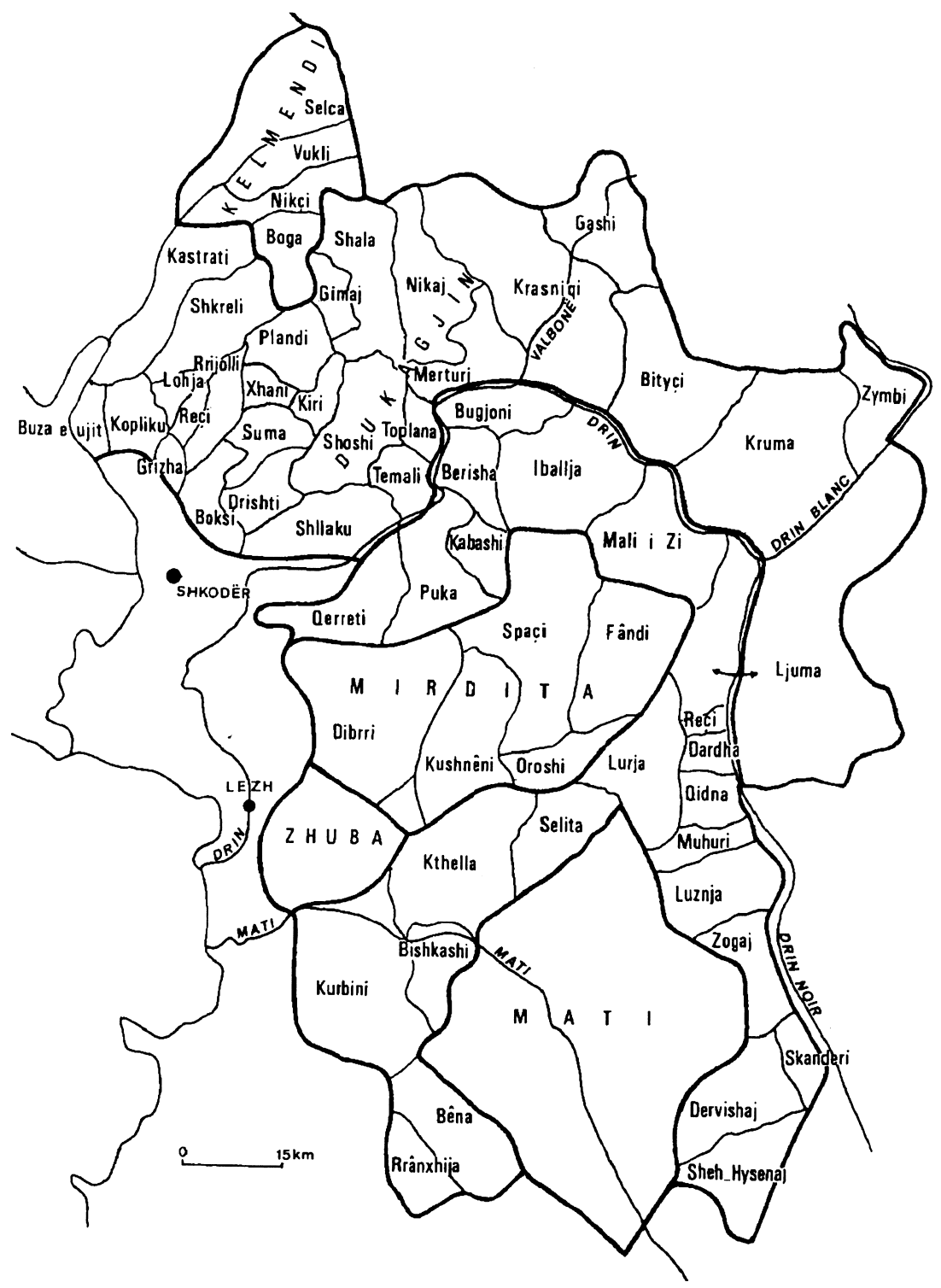

Figure 1. Région lignagère en Albanie du $\mathrm{N}$ ord. 
vllazni, ou 'phratrie', nom collectif formé à partir de vllazën, pluriel de vlla, 'frère'. Chaque këmbë est divisé en plusieurs vllazni, qui se divisent à leur tour en d'autres phratries d'un niveau inférieur en descendant les générations. La catégorie de vllazni apparaît comme un principe de segmentation dynamique regroupant à chaque niveau de génération les frères et leurs descendants dans une seule catégorie sociale. Une vllazni inférieure est toujours emboîtée dans une vllazni supérieure, tandis que au plus haut de la pyramide se situent le këmbë et le fisi. Cependant chaque vllazni apparaît comme un groupement à part entière reconnu par le nom de l'ascendant immédiat. Elle a plusieurs types de correspondants territoriaux hiérarchiquement déterminés. Mais elle entretient avec eux les mêmes rapports que le fisi et le këmbë avec leurs territoires respectifs. Par contre, le fisi et le këmbë sont des catégories statiques et symboliques, tandis que la vllazni est une catégorie dynamique et réelle. De ce fait, elle se présente comme une unité beaucoup plus importante que les deux premières, notamment en matière de stratégie matrimoniale et politique ou idéologique et religieuse. Cette relative indépendance stratégique de la vllazni peut expliquer en partie les différences dans l'appartenance religieuse d'une population organisée par ailleurs selon un même système de parenté demeuré fondamentalement identique (Doja 1998: 239-89).

Chaque vllazni est divisée non seulement en plusieurs autres vllazni hiérarchiquement dépendantes et qui portent le nom de leurs ascendants immédiats respectifs, mais

\begin{tabular}{|c|c|c|c|c|c|c|c|c|}
\hline No & Lignages & Membres & No & Lignages & Membres & No & Lignages & Membres \\
\hline 1 & Boga (Kelmendi) & 228 & 23 & Dervishaj & 995 & 45 & Plandi & 980 \\
\hline 2 & Nikçi (Kelmendi) & 685 & 24 & Drishti & 1,202 & 46 & Puka & 1,525 \\
\hline 3 & Selca (Kelmendi) & 852 & 25 & Gashi/Shipshani & 3,628 & 47 & Qerreti & 2,080 \\
\hline 4 & Vukli (Kelmendi) & 712 & 26 & Gimaj & 565 & 48 & Qidna & 1,338 \\
\hline 5 & Matja & 23,643 & 27 & Grizha & 1,044 & 49 & Reçi (Dibra) & 1,429 \\
\hline 6 & Piprri (Mirdite) & 5,774 & 28 & Iballja & 3,197 & 50 & Reçi (Shkodra) & 1,414 \\
\hline 7 & Fandi (Mirdite) & 3,332 & 29 & Kabashi & 1,494 & 51 & Rranxha & 423 \\
\hline 8 & Kushneni (Mirdite) & 2,430 & 30 & Kastrati & 3,280 & 52 & Rrijolli & 1,530 \\
\hline 9 & Oroshi (Mirdite) & 1,460 & 31 & Kiri & 534 & 53 & Selita & 1,877 \\
\hline 10 & Spaçi (Mirdite) & 4,230 & 32 & Kopliku & 1,455 & 54 & Shala & 2,512 \\
\hline 11 & Bulgri (Zhuba) & 769 & 33 & Krasniqi & 4,803 & 55 & Sheh-Hysenaj & 1,760 \\
\hline 12 & Kryeziu (Zhuba) & 897 & 34 & Kruma & 3,874 & 56 & Shoshi & 2,688 \\
\hline 13 & Manatia (Zhuba) & 629 & 35 & Kthella & 3,952 & 57 & Shkreli & 1,293 \\
\hline 14 & Velja (Zhuba) & 840 & 36 & Kurbini & 2,209 & 58 & Shllaku/Mazreku & 2,023 \\
\hline 15 & Bena & 1,643 & 37 & Luma & 17,978 & 59 & Skandri (Homesh) & 2,773 \\
\hline 16 & Berisha & 1,013 & 38 & Lohja & 709 & 60 & Suma & 641 \\
\hline 17 & Bishkashi & 2,191 & 39 & Lura & 1,605 & 61 & Temali/Dushmani & 939 \\
\hline 18 & Bytyçi & 2,044 & 40 & Luzna & 1,415 & 62 & Toplana & 254 \\
\hline 19 & Bokshi & 1,489 & 41 & Malizi & 2,528 & 63 & Xhani & 534 \\
\hline 20 & Bugjoni & 1,198 & 42 & Merturi & 2,211 & 64 & Zogaj & 1,656 \\
\hline 21 & Buza e Ujit & 995 & 43 & Muhuri & 1,304 & 65 & Zymbi & 11,140 \\
\hline 22 & Dardha & 1,198 & 44 & Nikaj & 1,652 & \multicolumn{2}{|c|}{ Total } & 160,695 \\
\hline
\end{tabular}

Figure 2. Densité et répartition religieuse de la population dans l'aire lignagère du $\mathrm{N}$ ord en 1918 (Source: Seiner 1922; cartographie: D aniel 1989). 
surtout en plusieurs maisons (shpi, shtëpi), qui constituent les groupes domestiques de base. Lorsque le groupe domestique devient trop nombreux et que la place ou les ressources sont insuffisantes pour loger et nourrir tous les membres, il peut 'éclater'. Après la mort du père, les tensions entre les frères mariés peuvent les obliger à se séparer pour s'installer ailleurs. La communauté formée des frères séparés et de leurs descendants s'appelle néanmoins toujours vllazni, le sens de 'fratrie' et celui de 'phratrie' devenant ainsi homologués.

Au total, l'organisation lignagère albanaise apparaît sous une forme quasi segmentaire et, de ce fait, elle est plutôt refermée sur elle-même. L'existence des groupements lignagers structure les rapports sociaux au sein d'un territoire donné. Pour résoudre la contradiction entre principe de parenté et principe de résidence, notamment dans les régions du Nord où l'organisation lignagère est attestée, les Albanais ont mis en œuvre un système de nomination spécifique incorporant tout individu, en un emboîtement progressif, dans les générations ascendantes et les unités de référence (fis-i, këmbë, vllazni, shtëpi) organisées de façon segmentaire. L'organisation lignagère s'est ainsi perpétuée au-delà même de son fonctionnement réel.

Dans certaines régions du Nord, la situation peut se comparer à celle que Robin Fox a observé dans l'île Tory en Irlande, où les habitants sont désignés par une chaine de prénoms, tous pris dans un stock familial, dont la combinaison permet de situer chacun dans son réseau généalogique et dans sa génération. ${ }^{1}$ De même, dans les Highlands d'Ecosse, jusqu'au dix-huitième siècle, la plupart des habitants portaient des noms généalogiques mentionnant les noms individuels des ascendants de plusieurs générations: A laistair M ac T earlach M ac Angus M ac I an D hu (Vroonen 1967: 79).

Chez les Albanais du Nord, outre son nom individuel, chaque individu se désignait traditionnellement par les noms du père, du grand-père, de l'arrière-grandpère, comme par exemple, G joka i M arka L lesh $\mathrm{N}$ due $\mathrm{N}$ dojt dans la région de Mirdita, ou bien Prenga i D od L lesh Per M ar G jetës dans la région de Kashnjet (Zojzi 1977: 195). On réunissait ainsi en son nom plus de six ou sept générations pour se rapprocher le plus possible du premier ancêtre du lignage. On pouvait utiliser aussi le nom de ce premier ancêtre comme nom patronymique. Mais dans les situations cérémonielles et ritualisées, la forme de référence la plus explicite pour nommer et être nommé restait celle qui marquait une suite ininterrompue d'indications patronymiques ('fils de...', souvent avec ellipse de 'fils' et le nom du père au génitif), remontant toutes les générations jusqu'au premier ancêtre. Dans la région de Hoti, par exemple, un individu se nommait Kolë Pashku i Pashk M arkut t'M ark Zefit t'Z ef $M$ arashit t'M arash U cit $t^{\prime} U$ c M ehmetit $t^{\prime} M$ ehmet $G$ jonit $t^{\prime} G$ jon L ekës t'L ekë $M$ arashit $t^{\prime} M$ arash Brulës t'B rulë Vuksanit t'Vuksan Pepës t'Pepë C ekut t'C ekë Jungjit t'Jungj G egës t'G egë Lazrit $t^{\prime} L$ azër K eçit t'K eç Pantës. Là on s'arrêtait, arrivé au nom du premier ancêtre dont tous étaient descendus (Zojzi 1977: 195).

La passion généalogique reste pourtant limitée dans la société albanaise. Sans doute parce qu'elle est inscrite toute entière dans l'ensemble des représentations sociales, dont le système de nomination est l'un des moyens de symbolisation les plus

1 'Dans la branche Eoin-Neilé, il y a une femme que j'ai toujours connue, comme tous le monde, sous le nom de Nora Thomais, "Nora fille de Thomas". Quand sa position dans la généalogie fut discutée, alors un interlocuteur nous étonna en récitant son nom complet: N ora-Thomais-JohnJohn-Eoin-N eilé (Norahomishjohnhohnianelly). Dit d'une seule traite, à toute vitesse, cela est impressionnant, mais incompréhensible sans autre explication.' (Cité dans Zonabend 1986: 28). 
efficaces. Chaque groupement (fis-i, këmbë, vllazni, shtëpi) encadre des situations déterminées d'un point de vue à la fois social et référentiel. La maison constitue le groupe domestique de base. C'est une instance statutaire des stratégies matrimoniales. Elle possède aussi les champs et les moyens de production. Elle apparaît donc comme une unité économique, politique et sociale à part entière. Il est clair que dans l'ensemble du monde indo-européen les termes désignant la 'maison' ont une valeur exclusivement sociale, sans qu'il y ait entre cette notion et celle de 'bâtir' ou d' 'habitat matériel' une autre relation que celle de contamination (Benveniste 1969: 1, 293-319). Mais il est remarquable qu'en albanais le terme shpi, shtëpi qui désigne la 'maison' ne signifie pas une simple notion d'habitation ou d'établissement du groupe social qui lui correspond. S'il s'agit d'un ancien emprunt au latin, il n'est pas construit à partir de casa ni de mansio, comme dans la plupart des langues romanes. Le mot sh(të)pi répond directement à hospitium en latin, ce qui dénote une valeur forte de relation sociale à travers la notion sous-jacente de l' 'hospitalité'.

La maison est subordonnée à un personnage central, zoti i shtëpisë, le 'maitre de maison'. Cette notion correspond sémantiquement à l'indo-européen *dems poti, qu'on transpose de la même façon dans les termes de la culture moderne par la traduction usuelle 'maître de maison'. Le second terme *pot(i)- a un sens à valeur forte, uniformément distribué dans les langues indo-européennes, qui se définit bien comme 'maître', avec un développement propre au latin et à l'italique vers la notion de 'pouvoir', d'où 'époux' dans la conjugalité, ou 'chef' d'une certaine unité sociale, maison, phratrie, lignage, clan, tribu. Mais il y a homophonie entre ce terme et une particule d'identité formée de la même manière, dont le sens est aussi bien attesté dans les langues indo-européennes et qui signifie 'même, self, soi-même'. Il est difficile de concevoir comment un mot désignant le 'maître' a pu s'affaiblir jusqu'à signifier 'luimême', mais on comprend d'autant plus facilement comment un adjectif marquant l'identité de la personne, signifiant 'lui-même', a pu assumer le sens propre de 'maître'. En fait, le maître de maison n'est autre que le ipse de la maison, l'être même de la famille, celui qui personnifie la maison en tant que cellule sociale, qui assume la personnalité et l'identité totale du groupe familial, au point de le résumer en lui-même et à lui seul de l'incarner (Benveniste 1966: 301-7).

Effectivement chez les Albanais, le rôle du zoti i shtëpisë, 'maître de maison', est moins d'exercer un pouvoir quelconque que d'assurer une représentation, plus particulièrement dans les assemblées communes. Il est celui qui agit au nom de la maison, et notamment le 'maître de l'hospitalité' offerte par sa maison. Ce n'est pas le pouvoir exercé mais le droit de représentation qui lui donne autorité sur l'ensemble familial avec lequel il s'identifie. L'individu qui fait partie de la maison portera le nom de celle-ci, qui est également son nom de famille. Ce nom peut coïncider avec son patronyme (nom individuel $\mathrm{du}$ père) si son père est le représentant légal dans les assemblées communes, autrement dit s'il est zoti i shtëpisë, le 'maître de la maison'. En tout cas, le nom d'un individu affiche ainsi trois éléments: son propre nom, le nom individuel de son propre père et le nom du maître de sa maison.

Au niveau immédiatement supérieur de la segmentation, la vllazni possède un même patrimoine foncier, essentiellement des forêts et des pâturages. Elle intervient sur le plan de la coopération économique et de l'entraide sociale, dans les activités cérémonielles, au moment des cérémonies du cycle de vie et d'année. Elle est dotée aussi d'institutions politiques, juridiques et militaires, qui souvent lui sont déléguées par le këmbë ou le fis-i. La D jelmeni, qui désigne littéralement la 'jeunesse', formait 
notamment le corps armé de la communauté lignagère. Mais indépendamment du nombre d'hommes dont elle peut disposer, la maison ne fournit à la D jelmeni qu'un seul homme armé, à qui elle délègue la 'propriété' de représentation du 'maitre'. En règle générale, celui qui assume cette fonction n'est autre que son fils aîné. Dans la société traditionnelle existe un véritable système de relations complémentaires et compensatoires. Chaque groupe social maintient ou reconstitue son unité dans toutes les circonstances où la société entière est engagée. Pour triompher dans cette grande épreuve que constitue le combat et qui est l'affaire de tous, l'organisation de l'armée doit se conformer aux cadres de la société. Ainsi elle aura sa plus grande efficacité.

S’agissant des principes de commandement, la parenté se prolonge encore dans la sphère du politique. L'opposition des aînés et des cadets se traduit dans les terminologies et joue un rôle fondamental dans le domaine de l'organisation. Les aînés de la génération d'Ego sont placés sur le même registre que les cadets de la génération de son père (le frère aîné d'Ego est comme un frère cadet du père) alors que les cadets de sa génération sont refoulés au niveau de la génération de ses fils (le frère cadet d'Ego est comme un fils). Il est donc logique que le pouvoir revienne de droit au chef d'une phratrie aînée, repérable par son nom. Cette réalité dans les régions du Nord est assez complexe car elle n'est pas déterminée en fonction du critère biologique de l'âge, mais comme il apparât dans le droit coutumier Kanuni i L ekë Dukagjinit, ${ }^{2}$ par la catégorie juridique de la primogéniture des ascendants, c'est-à-dire par le statut social d'aîné dans la branche aînée, transmissible d'une génération à l'autre (Gjeçov 1989: 60):

\begin{abstract}
D jalit të parë i përket sundimi i shpisëmbas dekës së t'et. Vëllau i parë do të pvetet për gjithshka brenda e jashta shpisë. D jalit të parë të vëllaut të parë, me kanë flamurtari, i përket flamuri, me kanë derë vojvodet, i përket prija, mekanëplak katundi, i përket strapleqnia.
\end{abstract}

Au fils aîné appartient l'autorité dans la famille après la mort de son père. L'avis du frère aîné est sollicité à chaque fois dans les affaires touchant la famille de près ou de loin. Au fils aîné du frère aîné, s'il hérite la bannière, il lui appartient la bannière, s'il hérite le commandement, il lui appartient le commandement, s'il hérite la chefferie, il lui appartient d'être le chef.

La D jelmeni, en l'occurrence, était organisée en détachements hiérarchiques qui reproduisaient l'organisation de l'ensemble de la société. Elle était dirigée par une hiérarchie de chefs qui héritaient leur fonction du fait d'être les premiers-nés dans la branche respectivement deuxième née de la vllazni, du këmbë et du fiśi. Aux premiers-nés dans les branches aînées respectives était théoriquement réservée la fonction de chef nominal de leur unité d'appartenance, alors que les premiers-nés dans la troisième branche héritaient de la fonction de bajraktar, 'porte-drapeau'.

Soient, par exemple, trois këmbë ou vllazni, descendant du même ancêtre fondateur, numérotés de 1 à 3 , suivant leur rang de naissance. Leurs représentants se répartissent la fonction du 'chef', qui exerce la représentation lui donnant autorité sur

2 Les normes juridiques traditionnelles les plus connues parmi les droits coutumiers albanais sont celles dénommées Kanuni i L ekëD ukagjinit, que la tradition fait remonter au prince Leka Dukagjini, compagnon de Skanderbeg, sans qu'on puisse confirmer la validité de cette relation. Elles ont été recueillies dans les régions du Nord et codifiées par le prêtre catholique Shtjefen K. Gjeçov, de Janjevo (Cossovie), à la fin du dix-neuvième siècle et au début du vingtième siècle. Son recueil posthume fut publié par les soins des Fransiscains de Shkodra en 1933. (Une deuxième édition, augmentée de manuscrits inédits, ne sera publiée par les soins de l'Académie des Sciences d'Albanie 
l'ensemble du groupe social (1), le commandement dans les opérations militaires (2) et le port du drapeau (3). À la génération suivante, trois fils descendant du premier-né de l'ancêtre fondateur, 1-1,1-2 et 1-3, vont se répartir de la même manière les mêmes fonctions: la représentation politique et juridique (1-1), le commandement dans les opérations militaires (1-2) et le port du drapeau (1-3). Et ainsi de suite pour les trois descendants du deuxième $(2-1,2-2,2-3)$ et du troisième fils $(3-1,3-2,3-3)$ de l'ancêtre fondateur. (Le premier chiffre exprime le rang de naissance et la deuxième le type de fonction.) Dans tous les cas, chacun a théoriquement la pleine jouissance de ses fonctions envers l'ensemble de sa descendance directe. Mais si les trois descendances en question reconnaissent effectivement l'appartenance au même ancêtre fondateur, qu'il s'agisse d'un fis-i, d'un këmbë, ou d'une vllazni, la hiérarchie des pouvoirs devient plus complexe. Si 1 délègue ses pouvoirs à 1-1,1-2 et $1-3$ sur l'ensemble de la communauté lignagère, 2 ne délègue ses pouvoirs à $2-1,2-2$ et $2-3$ que sur la descendance directe et l'ensemble des descendances inférieures par rapport à son rang de naissance, c'està-dire sur la descendance 2 et sur la descendance 3 . De cette façon, 1-1, 1-2 et 1-3 sont hiérarchiquement supérieurs non seulement envers $2-1,2-2$ et $2-3$ ou envers $3-1$, $3-2,3-3$, mais aussi envers 2 et 3 qui appartiennent pourtant à une génération antérieure. Toutefois, ce type du partage des pouvoirs ne fut jamais vrai qu'en théorie. Avant même la décomposition définitive du système segmentaire à la fin du dixneuvième siècle, dans la plupart des cas, le bajraktar réussissait à concentrer dans ses mains l'ensemble des trois fonctions.

Pareil schéma a le mérite d'exprimer à sa façon la fonction hiérarchisante de l'âge. Sur la base de ce principe, tout le monde était en état de se ranger même en grand nombre en respectant le rang et la place qui revenait à chacun dans les différentes occasions rituelles, par exemple, prendre la parole aux assemblées et aux réunions, faire la queue pour se laver les mains, se mettre à table, aller en procession nuptiale, etc. (Zojzi 1977: 197).

Pour marquer ce type d'appartenance dans une situation donnée, l'individu affiche son propre nom, le nom du maître de sa maison et en troisième position le nom du représentant de la phratrie, qu'il considère cette fois comme son véritable nom de famille. La phratrie étant par définition une catégorie très mouvante, le troisième élément d'un nom de personne, que nous appellerons volontiers nom de phratrie, change constamment pour se référer, selon les situations, à différents niveaux de la chaîne segmentaire entre la maison et le këmbë du lignage.

L'adoption en troisième position du nom de l'ancêtre fondateur du këmbë ou du fisi, catégories à la fois plus stables et plus discriminantes, est réservé aux usages extérieurs aux territoires d'influence et aux espaces de référence du këmbë et du fisi. En deuxième position, toute personne a alors le choix entre le nom de sa propre maison et l'un des noms de phratries qui se situent aux niveaux supérieurs intermédiaires avant le

qu'en 1989, entretemps il fut publié en italien et en allemand). La plupart des travaux ethnographiques dénomment du terme de Droit de Leka Dukagjini les normes juridiques coutumières observées dans la région de Dukagjini en Albanie du Nord et sur le Plateau de Dukagjini jusqu'en Cossovie (province autonome de la Serbie actuelle), c'est-à-dire sur le territoire des anciens domaines de la principauté des Dukagjini. Mais dans la tradition albanaise, les droits de la coutume s'étendaient sur un domaine plus large, notamment dans ces régions où ont été repérés les derniers vestiges de l'organisation lignagère de type segmentaire. En Albanie centrale, sur la rive droite de Shkumbini, dans la région de Ghèghenie proprement dite, où s'étendaient les anciens domaines de la principauté de Georges Kastriote, dit Skanderbeg, les travaux ethnographiques ont retenu des normes semblables 
terme de référence utilisé en troisième position. En Irlande aussi, certains individus portent encore deux noms de famille, qui peuvent être deux noms d'ancêtres différents ou deux désignations différentes du même ancêtre (Vroonen 1967: 82).

Ainsi, au moyen $\mathrm{du}$ nom, tout individu a possibilité de s'ajuster à telle ou telle situation concrète. Ces variations dans la nomination sont dues à la relativité des 'valeurs de groupes' auxquels le nom renvoie. Il s'agit de la relativité des éléments dans un système segmentaire, où 'les subdivisions des divers ordres coexistent virtuellement à tout instant et ne se manifestent qu'alternativement dans des situations déterminées' (Dumont 1971: 55-8). Il faut donc comprendre cette relativité, pour que les fluctuations de nomination soient perçues comme une qualité intérieure à la structure même d'organisation sociale.

Effectivement, c'est surtout dans les régions du Nord qu'on trouve régulièrement des noms tripartis, tels que D ed Gjo Luli, Preng Bib Doda, Prelë M emë Ujka, mais parfois dans celles du Sud aussi, comme dans la région de Labëria: R ustem G jondedaj (R. Gjon Dedaj), I sa Gjonzeneli (I. G jon Zeneli), M etat G jonligu (M. Gjon Ligu), M uslim G joleka (M. G jon L eka), Ali G jovarfi (A. G jon Varfi). Ces noms de personne à trois éléments sont présents en Albanie depuis le haut Moyen Âge (Çabej 1939: 194-213). Ainsi Gjin Bua Shpata, le plus connu de la grande famille princière des Shpata au Sud, ou bien Tanush Topia, un autre grand nom de l'histoire médiévale en Albanie centrale, qui est appelé Tanush Muzak Topia dans une chanson populaire (Gurakuqi et Fishta 1937: 19). Les noms des héros des chansons narratives du Nord, G jeto Basho M uyi, Gjergj Elez Alia, Destar O sman Aga, Bur Eleze Krajli, Siran O sman Aga, Bud Aline Tali, Ager I svan Aga, $\mathrm{H}$ ake Vrak Shabani, comportent tous trois éléments. Dans le cycle des gestes de Muyi même les noms bipartis deviennent des noms tripartis par répétition d'un élément, comme Ali Borxh Alia, A ga $\mathrm{H}$ asan Agë, Beg-e M ehmet Begu, Begu Allaj Beg, Pasha $\mathrm{H}$ asan Pashë. Cette forme de nomination comprend le plus souvent le prénom, le nom individuel du père et un troisième nom évoquant un surnom ou une référence généalogique ou territoriale.

Les affaires de vengeance sont exemplaires pour illustrer le fonctionnement de cette organisation lignagère et de ce système de nomination tripartie de manière parfaitement segmentaire, ou structurale, dans le sens de Evans-Pritchard. L'appartenance des acteurs à l'un ou l'autre des groupements lignagers partageant un même territoire apparaît alors comme le ressort de l'action. Selon que les personnes concernées appartiennent à deux maisons de la même vllazni, à deux vllazni du même këmbë, à deux këmbë du même fisi ou à deux fisi différents, les modalités de vengeance seront différentes et le conflit impliquera des groupes de niveaux différents. Il peut impliquer soit des lignages entiers, si un homme a été tué par un homme d'un autre fisi, soit seulement deux maisons, si le meurtrier et la victime appartiennent à la même vllazni. A terme, la réconciliation est possible à l'intérieur d'un lignage, mais plus rare entre deux lignages.

Ce système met souvent en contradiction les relations de parenté et les stratégies de relations, qui ne peuvent être que définies d'avance, en fonction de la position

sous le nom de Kanuni i Arbërisë 'Droit d'Albanie' ou Kanuni i Skënderbeut 'Droit de Skanderbeg'. Dans d'autres régions, notamment dans la région des Montagnes d'Albanie du Nord depuis la Montagne de Shkodra jusqu'à la Montagne de Gjakova et la Plaine de Cossovie c'est le terme de Kanuni i Maleve 'Droit des Montagnes', qui a été retenu. Il est cependant fort probable que ces dénominations locales soient des variantes des mêmes normes juridiques coutumières, observées 
structurelle occupée par chacun au sein du système. Il semble évident que pour satisfaire à la nécessité de s'ouvrir aux 'autres' et de tisser des relations indépendantes, cette structure d'organisation sociale paraît trop rigide et insuffisante. Par conséquent, il doit y avoir nécessairement d'autres catégories d'organisation sociale, telles que l'alliance et l'affinité, qui permettent notamment une plus ou moins grande souplesse selon les stratégies d'ouverture et de communication du groupe social.

\section{Le modèle linéaire: affinité de parentèle}

Dans la liste des différents types de groupes issus de la segmentation on peut également trouver le terme barku, littéralement 'ventre, matrice', dans le sens de 'progéniture, lignée portée et engendrée par le ventre d'une femme'. Rrok Zojzi a considéré ce terme comme une catégorie intermédiaire entre la phratrie et la maison (1977: 185-206). Il faut noter qu'il est souvent remplacé par gjiní (gjirí) ou gjení (gjerí). Le mot, dérivé du grec byzantin $\gamma \in \nu \in \alpha$ (Çabej 1996: 332), se rattache à gens en latin, à génos en grec et à l'intérieur de l'albanais à gjíni 'sein, poitrine', qui révèle une valeur forte liée à la maternité et à l'allaitement, comme dans fëmijë gjíni 'nourrisson'. Le fait important est que le sens de ces termes dépend étroitement du sens de la racine commune indoeuropéenne *gen- qui indique la 'naissance'. Dans une organisation sociale définie par les groupements segmentaires, la naissance est en effet une condition du statut personnel. Plusieurs dérivés nominaux dans les langues indo-européennes, analysés par Benveniste, mettent en évidence que du sens premier de la naissance physique on est passé à la naissance comme fait social. Ce sont des termes qui, en la dénommant, caractérisent la naissance comme légitime, en ce qui concerne les droits qu'elle confère à ceux à qui elle est reconnue. En outre, la légitimité de naissance vaut d'abord pour les hommes. C'est aux hommes que s'appliquent les dérivés collectifs tirés de la racine indo-européenne, en désignant ceux qui se reconnaissent un ancêtre commun (1969: 1, 315-8).

Mais la dimension du groupe social que chacun de ces termes désigne peut varier selon les sociétés. Ils n'occupent pas la même place dans la nomenclature des divisions sociales. Si en grec et en latin ces dérivés désignent généralement la descendance masculine en lignée paternelle, en albanais c'est toujours la descendance masculine que le terme gjiní désigne, mais essentiellement en lignée maternelle. Le terme albanais semble ainsi avoir conservé plus longtemps le sens antique de la naissance physique, qu'on peut retrouver de façon plus manifeste dans lisi i tamlit 'l'arbre du lait', de la même façon que dans barku 'ventre, matrice', équivalents de gjiní 'lignée maternelle'. C'est précisément dans ce sens que le terme gjiní est alors opposé à gjaku, littéralement le 'sang', qui désigne la lignée paternelle dans l'expression lisi i gjakut 'l'arbre du sang' ou bien dans l'expression gjak e gjiní (gjak e gjirí) utilisée pour signifier la parenté indifférenciée. Les deux termes ne désignent pas des unités segmentaires socialement constituées, mais plutôt une reconnaissance réelle de l'appartenance à la série linéaire paternelle ou maternelle correspondante, comme il apparaît clairement dans différents articles des normes coutumières du Nord (Gjeçov 1989: 51-2):

depuis la vallée de Shkumbini jusqu'en Cossovie et probablement dans le du Sud-Ouest de l'Albanie, dans les régions de Vlora, Kurveleshi, Himara, Tepelena, généralement connues sous le nom de Labëria, jusqu'aux Montagnes des Souliotes. Dans ces régions du Sud-ouest, c'est encore un autre terme, K anuni i L abërisë 'Droit de Labëria', qui a été retenu par les travaux ethnographiques. 
Vargu i gjatë i brezave të gjakut e të gjinísë shkon nëtëpasosun për shqyptarin emaleve. Brezat rrjedhin a prej gjakut a prej gjinísë. Brezat e gjakut rrjedhin prej anet të babës, brezat e gjinísë prej anet të nanës.

R rjedhja ebrezave prej anet tëbabësthirret ' lisi i gjakut' ; rrjedhja ebrezave prej anet të nanës thirret 'lisi i tamlit'.
La longue chaîne des générations de gjaku et de gjiní va à l'infini pour l'Albanais des Montagnes.

Les générations découlent de gjaku (sang), ou de gjiní. Les générations de gjaku émanent du père, les générations de gjiní émanent de la mère.

La filiation patrilinéaire s'appelle lisi i gjakut, 'l'arbre du sang', la filiation matrilinéaire s'appelle lisi i tamblit, 'l'arbre du lait'.

Un individu a donc de fait deux catégories de parents: ceux qui viennent de son père, 'l'arbre de sang', et ceux qui viennent de sa mère, 'l'arbre de lait'. Ce principe de descendance constitue l'axe de tout le système et devient porteur d'une double référence. Elle privilégie ici la distinction entre sang et lait, qui semble être une transposition de la classification, plus familière aux ethnologues, entre le 'côté de l'os' et le 'côté de la chair' (Lévi-Strauss 1967: 430). L’idéologie albanaise reste au demeurant fortement patrilinéaire et il ne s'agit pas là d'un principe de descendance cognatique où le père et la mère interviendraient à égalité. L'arbre de sang implique toujours une certaine bilatéralité mais conserve sa supériorité en valeur. Le cognatisme n'est qu'apparent. En partant de la croyance que l' 'os', ou ses équivalents, le 'sang' en l'occurrence, vient du côté du père et la 'chair', ou le 'lait' comme équivalent, du côté de la mère, ce système de représentations situe pour Lévi-Strauss non pas 'des individus - père et mère - qui contribueraient chacun pour leur part au corps de l'enfant, mais des groupes, ou des lignées, dans leur rapport de coopération dans et par l'alliance matrimoniale' (1967: 454).

Les recherches sur la parenté ont peut-être un peu trop considéré l'alliance dans un schéma d'extériorité dualiste, ne laissant pas suffisamment voir la présence conjointe des parties contractuelles elles-mêmes. Procédant de logiques opposées, les alliés se définissent couramment par rapport à Ego. Ainsi, ses parents par le lait ne sont considérés que comme les alliés de son père. Bien que les catégories ne s'opposent pas en contenu, puisqu'elles représentent les mêmes gens, il s'agit de deux catégories différentes. Les données empiriques sont assez explicites pour ne pas se rendre compte que, lorsque ce groupe de gens est défini en fonction d'Ego, on a affaire à la filiation et non pas à l'alliance. Ce n'est que lorqu'ils sont définis par rapport au père d'Ego, qu'on peut parler d'alliance. Cette même confusion a eu pour effet de faire représenter l'alliance comme un point d'intersection normale entre la lignée paternelle et la lignée maternelle d'Ego.

Les données empiriques de provenance albanaise témoignent clairement que les parents par le lait d'Ego constituent l'arbre de sang (gjaku) de l'oncle maternel. Au moment du mariage du père d'Ego, ce 'sang' s'incarnait dans l'arbre de lait (gjiní), c'està-dire dans les parents par le lait d'Ego. De la même façon, Ego n'a pas le même sang que ses neveux utérins. À son tour, lorsque la sœur d'Ego se mariera, elle n'apportera pas de parents par le sang à ses enfants. Les parents par le sang leur seront apportés par leur père, mari de la sœur d'Ego. Elle apportera à ses enfants des parents par le lait, dont le principal, Ego, représente un arbre de sang (gjaku) qui devient allié de l'arbre de sang (gjaku) du mari de sa sœur. Ainsi, Ego appartient au même arbre de lait (gjiní) que les enfants de sa sœur, et ses enfants appartiennent à l'arbre de lait (gjiní) de sa femme. Ego et ses enfants appartiennent au même arbre de sang (gjaku), tandis que les enfants de sa sœur appartiennent à l'arbre de sang (gjaku) du mari de celle-ci.

Il s'agit avant tout de repérer un autre 'sang' du côté du 'lait', celui-ci n'étant pris en considération qu'en fonction du 'sang' qui le définit. En d'autres termes la notion de l'arbre de sang, qui est la part manifeste de l'opposition entre sang et lait, est centrée sur 


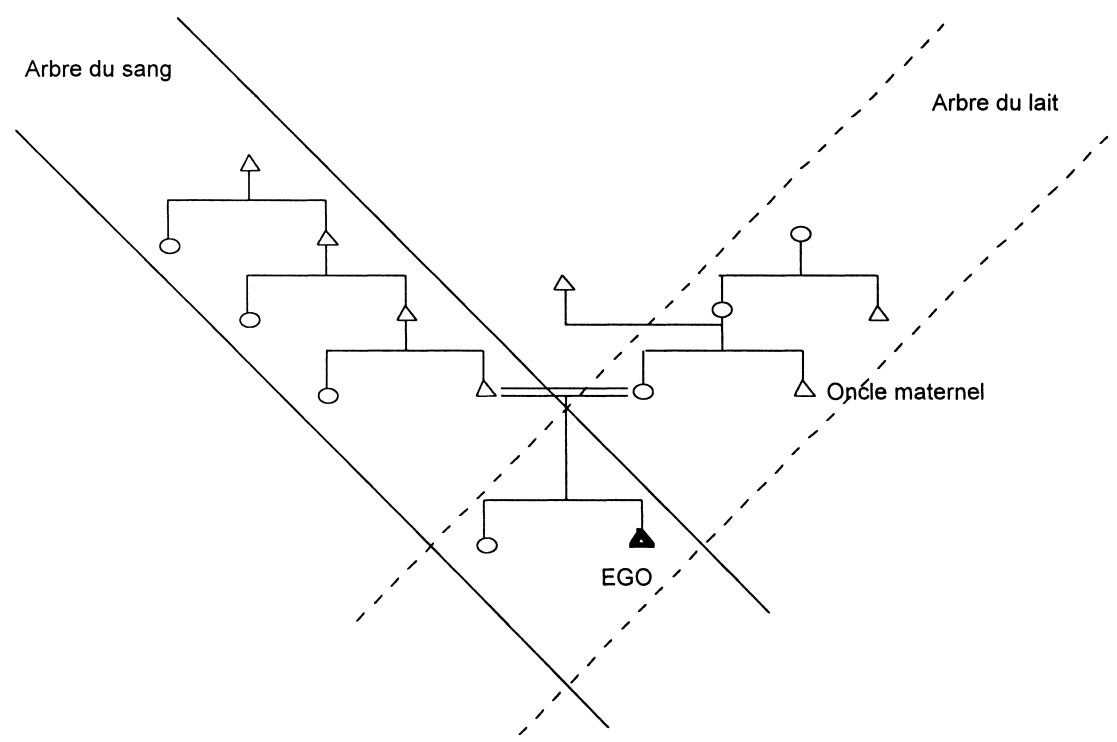

Figure 3. La rencontre orthogonale d'un arbre de lait et d'un arbre de sang ne traduit pas l'alliance matrimoniale, mais les deux lignées convergentes des ascendants paternels et maternels d'E go.

le 'sang' constitutif de la lignée. Dans cette représentation de l'alliance matrimoniale qui laisse supposer l'existence d'un système d'échange généralisé, chaque groupe est à la fois arbre de sang (gjaku) et arbre de lait (gjiní), comme les individus qui le constituent. Il est arbre de sang (gjaku), par rapport à la filiation d'un ascendant masculin, comme receveur d'épouses. Il est arbre de lait (gjiní) en tant que patrilignage donneur de mères. Mais ce double statut est toujours défini par rapport à des partenaires différents. Car dans un échange matrimonial de type généralisé, deux groupes donnés forment toujours l'un par rapport à l'autre un couple d'oppositions dans lequel un groupe est 'sang' (gjaku), et rien que cela, l'autre 'lait' (gjinì), et rien que cela (Lévi-Strauss 1967: 454-5). Alors que pour Ego, les deux lignées, paternelle et maternelle, sont certes des arbres de sang: son patrilignage et le patrilignage de sa mère. Mais ce dernier est réduit, centré sur le frère de celle-ci. De même, lors d'un échange matrimonial, l'arbre de lait, le groupe gjiní donneur d'épouse, est défini en tant qu'un arbre de sang, il est centré sur le 'sang' qu'il contient, ce sang étant incarné par le frère de l'épouse. Ce qui compte chez les parents par le lait n'est pas le 'lait' en lui-même, mais le 'sang' qui l'accompagne. Au moment de choisir une épouse pour son fils, un homme se renseignera sur le frère de l'épouse potentielle, ainsi que sur son oncle maternel, voire sur le grand-oncle maternel. Le système oppose ainsi deux pôles lignagers, le père et l'oncle maternel, l'oncle paternel n'ayant qu'un statut de frère aîné d'Ego.

La notion de l'arbre de sang exprime moins une relation agnatique ou cognatique que la complémentarité entre les deux lignées partenaires mais inégales en statut. L'une, lignée paternelle, s'appuie sur les valeurs de son 'sang' dans toute stratégie sociale, l'autre est un groupe donneur de 'lait nourricier', qui sert à fortifier les valeurs du 'sang' par la transmission d'un autre 'sang' venant des mères. En effet, le manque du lait maternel, l'absence d'allaitement au sein de sa propre mère, sont toujours considérés comme de grands malheurs lourds de conséquences dramatiques, non seulement individuelles, mais parfois collectives et sociales. 
On croyait que la destinée future de l'enfant, le développement de sa personnalité et ses réussites sociales dépendent de la qualité du lait maternel. Tant que dure l'allaitement, la relation entre la mère et l'enfant est privilégiée et autant de qualités ou de défauts, physiques comme morales, retransmis par le lait, peuvent s'imprimer de façon indélébile sur la physionomie et le caractère de l'enfant. Si dans l'avenir il mène à bien ce qu'il entreprend, le mérite revient à la mère. L'un des serments et des vœux les plus importants chez les Albanais porte toujours sur le lait maternel: Për qumësht të nënës! 'Pour le lait de la mère', H allall qumshtin e nënës! 'Béni soit le lait de la mère', et chez les habitants des Montagnes du Nord sur les seins maternels même: Për gjinjtë e nënës! 'Pour les seins de la mère', Pafsh gjinjtë e nënës! 'Au vu des seins de la mère'.

Une connotation forte est induite par l'importance de l'allaitement maternel à cette même relation de parenté aussi immédiate que la notion de 'fils'. Du point de vue indo-européen, cette notion est exprimée par des termes qui, avant de passer dans la nomenclature de la parenté, se sont rapportés à la notion d' 'enfanter' ou de 'rejeton, progéniture'. Mais une innovation commune au latin, au letton et à l'albanais, remarquée par Antoine Meillet (1920: 45-48), implique la notion de 'nourrir' et plus précisément de 'nourrisson allaité à la mamelle'. D'autant plus que le parallèle albanais correspond au mot djalë, 'garçon', mais aussi 'rejeton, progéniture', qui reflète encore aujourd'hui de façon évidente la spécialisation de sens passant d'un terme de progéniture à un terme de parenté (Çabej 1987: 255-8). On pourrait dès lors porter cette importance immanente de l'allaitement maternel, puisqu'on le trouve dans la terminologie générique de la parenté désignant la progéniture humaine, chez d'autres peuples aussi, ayant des mots de même origine pour ces termes, par exemple, au français fils dérivé du latin filius.

Le fait d'avoir bu au sein de la même femme qu'un autre, dans la tradition albanaise, donne lieu à une forme de parenté qui peut même entraîner des interdictions de mariage. Bien que rarement mentionnée, elle est connue par toutes les populations sud-est européennes (Stahl 1977: 289). En Cossovie et dans les Montagnes du Nord, lorsqu'une vieille femme meurt, ses proches vont lui embrasser les seins nus avant l'enterrement. Dans la Montagne de Dukagjini au Nord, aussi bien que dans les régions reculées de Tomorri et de Labëria au Sud, les seins sont gravés sur les encadrements en pierre ou en bois des portes des maisons comme des symboles de fertilité. En Cossovie, on parle même d'un interdit de frapper la terre, de peur de frapper les seins de la mère morte (Tirta 1980: 79-85).

Dans ce culte albanais des seins maternels on a souvent cherché à reconnaître les vestiges d'une divinité chthonienne telle une Magna Mater (Poghirc 1987: 178-80). Beaucoup d'autres rituels sont attestés chez les Albanais, au Nord comme au Sud, qui rappellent les représentations du même culte. En Myzeqe, par exemple, si les conjoints ne sont pas en bonnes relations on leur donne à boire du lait aux seins de deux nourrices mère et fille. En Myzeqe également la mère donne à boire de l'eau à son fils ou à sa fille le jour de leur mariage de par un pli de sa chemise en les félicitant du vœu traditionnel: $H$ allall qumshtin që të kam dhënë !, 'Béni soit le lait que je t'ai donné !' La belle-mère aussi, à Mallakastra au Sud et à Has près de Kukës au Nord, quand elle reçoit la nouvelle mariée le jour du mariage, elle l'embrasse trois fois sur le front et sur les seins.

Ceci dit, dans la complémentarité nécessaire entre l'arbre du sang et l'arbre du lait, la dimension patrilinéaire manifeste (le sang) est néanmoins privilégiée contre ce qui vient du côté des maternels (le lait). Dans un contexte où chaque patrilignage est à la 
fois preneur et donneur de femmes mais vis-à-vis de partenaires avec lesquels la relation n'est jamais réciproque, tout se passe comme si le problème était de trouver un ancrage du côté des maternels qui sont 'le lait dont nous nous sommes nourris'. Mais pas de 'lait' sans 'sang'. Le 'sang' du 'lait', nécessaire à la relation de complémentarité, est le patrilignage issu de l'oncle maternel.

Les parents par le lait d'Ego s'agrègent ainsi en une ligne matrilinéaire qui n'est autre qu'une suite de points d'intersection des arbres de sang respectifs du père de la mère d'Ego, du père de la mère de la mère d'Ego, etc. Au bout du compte, il faudrait considérer l'alliance matrimoniale non pas comme la convergence orthogonale entre un arbre de sang et un arbre de lait, mais plutôt comme le rapprochement parallèle entre un arbre de sang et un autre arbre de sang, qui se voient partager, de ce fait, le même arbre de lait.

Le fait remarquable est que, chez les Albanais, si une relation d'alliance s'établit entre deux arbres de sang par un rapprochement parallèle au moyen d'un même arbre de lait partagé, une relation d'hostilité s'établit de la même manière entre deux arbres de sang différents à travers une distance conflictuelle, souvent forcée par la difficulté de partager un arbre de lait. Ceci peut être le résultat d'une dégradation, pour une raison ou une autre, des valeurs incarnées dans le sang. Le terme qui exprime la vengeance en albanais est de nouveau gjaku, le 'prix du sang', dans des vocables comme jam/bie në gjak, marr gjak 'être/tomber dans le sang', ou zoti i gjakut 'le maitre du sang'.

$\mathrm{Du}$ fait de leur filiation patrilinéaire, fisi, këmbë, vllazni et gjaku (arbre de sang) rassemblent les mêmes gens, tous issus du même ancêtre fondateur. Mais l'arbre de sang (gjaku) n'est pas une unité du système segmentaire comme fisi, këmbë, ou vllazni.

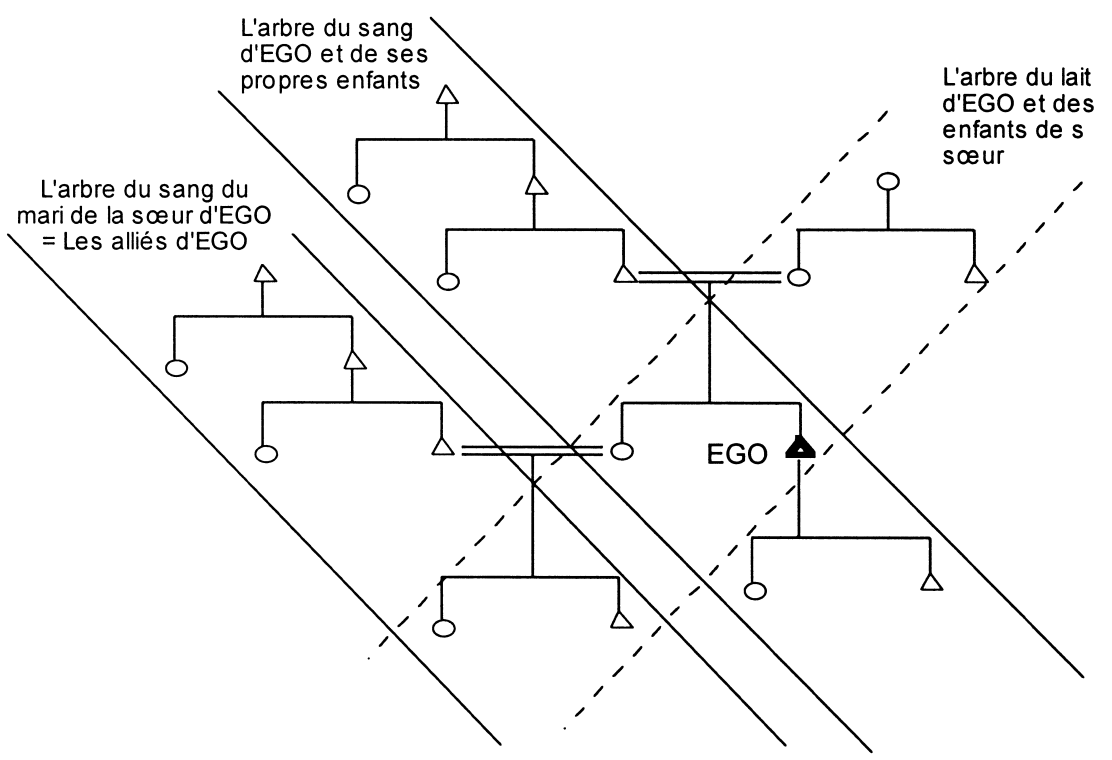

FiguRE 4. L'alliance matrimoniale est un rapprochement parallèle contracté entre deux arbres de sang qui se voient partager le même abre de lait.

3 Doja 1998: 'Amitié, alliance, parenté: idéologie et pratique dans la société traditionnelle albanaise', dans Georges Ravis-Giordani (ed.), A mitiés: anthropologie et histoire, Aix-en-Provence : Presses Universitaires de Provence, à paraître. 
Il n'est pas non plus disposé en fonction des références ancestrales. Il s'agit d'un patrilignage linéaire, centré sur Ego et inscrit dans un échange matrimonial, autrement dit une parentèle. Si les unités segmentaires de l'organisation lignagère ne sont attestées, en tant que catégories d'un système économique, politique, social et symbolique particulier, que dans certaines régions isolées au Nord de l'Albanie et peut-être dans une partie des régions du Sud-Ouest, la catégorie linéaire de l'arbre du sang (gjaku) est largement généralisée chez tous les Albanais. Elle est encore significative, même dans les agglomérations urbaines, dans les plaines et, d'une façon générale, dans les régions du Sud, réputées être moins sous le poids des traditions. Dans les régions du Nord même, elle reste plus fonctionnelle que les catégories segmentaires qui relèvent plutôt de l'ordre symbolique.

L'arbre de sang (gjaku) peut remonter chez les Albanais jusqu'à la septième génération et les cousins au septième degré, appartenant au même arbre de sang, sont des conjoints prohibés (Dojaka 1978: 73-8). L’arbre de lait (gjiní) permet en revanche de faire l'articulation entre parenté et système politique et idéologique. Deux groupes patrilinéaires qui partagent le même 'arbre de lait' (gjiní) sont en position d'alliés. Le code des normes coutumières règle leurs relations en matière d'alliance et de vengeance, d'entraide sociale et d'appartenance religieuse, d'amitié et d'hostilité. Les pratiques matrimoniales devront respecter les alliances politiques ou sociales entre groupes, avoir lieu de préférence avec des groupes déjà alliés politiquement et idéologiquement. Elles servaient souvent à créer cette alliance, en marquant la fin d'une période de conflit, qui pouvait aller souvent jusqu'à transgresser même les divisions religieuses nominales (Doja 1998: 239-89).

L'opposition entre arbre de lait et arbre de sang procède en effet comme une catégorie sociale qui permet l'ouverture transactionnelle dans des relations orientées vers l'autre. C'est en fonction de la référence aux valeurs du 'sang' qu'il semble possible de comprendre les 'grandes maisons' ou shtëpitë me emër, les 'maisons de grand nom'. La réputation de leur nom correspond à la notion de l'arbre du sang, défini par la descendance d'un sang commun, mais aussi par l'alliance avec un autre patrilignage ayant à sa tête le frère de la mère. Autrement dit l'arbre de sang désigne d'abord une maison et ses ramifications d'importance plus ou moins grande, en fonction des patrilignages avec lesquels elle est en relation comme partenaire immédiat d'une alliance matrimoniale. C'est, semble-t-il, selon l'étendue du groupe dominant, 'la grandeur de la maison', et selon l'importance de ses partenaires que la communauté gardera ou non la dignité de son nom.

On peut dès lors opposer deux formes de références: une forme typifiante segmentaire et holiste - et une forme classifiante - linéaire et individualisatrice. Catégories linéaires et catégories segmentaires ne s'opposent pas, puisqu'il s'agit toujours de filiation patrilinéaire. Mais contrairement au système segmentaire, la notion de lisi i gjakut, 'l'arbre de sang', en tant que catégorie linéaire, ne permet pas de remonter les générations. Si un grand nombre de parents situés collatéralement se reconnaissent appartenir au même arbre de sang, il leur faudra nécessairement un marqueur symbolique héréditaire, qui palliera le défaut de mémoire et/ou de segmentation à partir de l'axe vertical. La fixation du nom patronymique au sens large, le nom de famille transmissible, devient désormais nécessaire.

Effectivement, le système de nomination albanais s'est montré assez souple pour introduire à côté des noms d'ancêtres, des noms ethno-géographiques, des noms issus de génitifs pluriels désignant le plus souvent une appartenance territoriale et qui se sont 
singularisés et sont devenus des noms de famille, ou bien des noms d'attributs personnels, professionnels ou fonctionnels, des surnoms évoquant des caractéristiques individuelles ou territoriales, etc. (Boissin 1965: 176-82; Çabej 1966: 70-81, Krasniqi 1977: 377-415).

Dans les documents médiévaux, à côté de noms simples comme Gjin, Gjon, d'autres sont dotés d'un patronyme (nom de père), comme Ginus Tanusius, qui devient par la suite un nom de famille, comme Progon M ira. Il y a des noms de famille modifiés, provenant de noms ethniques ou de lieux, comme L azarus M alliocus, d'une activité sociale, comme Demetrius L allimi - de lajmës 'crieur, messager', d'un trait physique, comme Paulus Burmad - de burrmadhi 'homme grand de taille', Theodorus Këshetësi - 'qui porte les cheveux en tresse', d'un surnom, comme N icolaus D er, Georgius Passimada. Les noms de famille albanais peuvent provenir aussi de noms d'animaux utilisés d'abord comme des surnoms tels que Demi, 'taureau', Mazi, 'poulain', D ashi, 'bélier'. Ainsi les noms des grandes familles princières qu'on connaît dans l'histoire albanaise, comme les Bua - de buall 'buffle', les M uzaka - de mëzati 'poulain', etc. (Çabej 1960: 104).

Notre conviction est néanmoins que l'apparition du nom de famille a dû correspondre, au moins dans la société traditionnelle albanaise, notamment à la notion de l'arbre du sang, en tant que catégorie d'une organisation linéaire des rapports sociaux de parenté. La consolidation du nom patronymique a effectivement concerné d'abord les régions où l'organisation segmentaire a été de moins en moins pertinente. La contraction des noms tripartis en noms bipartis dans les régions du Sud illustre parfaitement cette évolution. Dans la région de Labëria au Sud, les trois noms se contractent en deux, M ehmet $\mathrm{G}$ jon Ligu devient désormais M ehmet $\mathrm{G}$ jonligu. Alors que dans les autres régions, où l'organisation segmentaire restait encore dominante, ne serait-ce que symboliquement, les noms tripartis se sont davantage conservés. Ils se sont même développés sans pourtant s'opposer aux noms bipartis qui, avec l'arrivée de la législation administrative, ont été érigés en nom de famille. Car la nomination en trois éléments, qui ne sont pourtant plus suffixés ni déclinés, a été adoptée par le système officiel en Albanie, quoique vidée de sa connotation segmentaire. Généralement considérée comme très spécifique à ce pays, cette forme de nomination témoigne de l'importance qu'attachent les Albanais à leurs liens territoriaux, parentaux et familiaux.

\author{
Albert D oja \\ 22 rue Emeriau \\ 75015 Paris \\ France
}

\title{
References
}

Baldacci, Antonio. 1917. I tinérari albanesi 1892-1902. Roma.

Benveniste, Emile. 1966. Problèmes de linguistique générale. Paris: Gallimard. 1969. L e vocabulaire des institutions indo-européennes, 2 vols. Paris: Minuit.

Çabej, Eqrem. 1939. 'Die albanische Volksdichtung', L eipziger Vierteljahresschrift für Südosteuropa 3, 3, 194-213.

1987. Studime etimologjike në fushë të shqipes. Etudes d'étymologie albanaise 3: C-D. Tirana: Académie des Sciences.

1996. Studime etimologjike në fushë të shqipes. Etudes d'étymologie albanaise 4: DH -J. Tirana: Académie des Sciences. 
Coon, Carleton S. 1950. The mountains of giants. A racial and cultural study of $\mathrm{N}$ orth Albanian mountain ghegs. Papers of Peabody Museum of American Archæology and Ethnology, 23, 3.

Cozzi, Ernesto. 1910. 'La vendetta del sangue nelle montagne dell'Alta Albania', Anthropos 5: 654-87.

1944. Le tribu dell'A Ibania, Roma: Istituto di Studi Albanesi, Serie giuridica.

Daniel, Odile. 1989. 'Montagnes tribales et coutumières', L'Ethnographie 85: 43-74.

Degrand, A. 1901. Souvenirs de la H aute A lbanie. Paris: Walter.

Doja, Albert. 1988. 'La relativité des idéologies religieuses dans les recompositions de l'identité albanaise.' Les Temps M odernes, 601: 239-89.

Dojaka, Abaz. 1978. 'L'exogamie chez les Albanais', Ethnographie Albanaise 8: 73-8.

Dumont, Louis. 1971. Introduction à deux théories d'anthropologie sociale. Paris: Mouton.

Durham, Mary Edith. 1909. H igh Albania, London: Arnold (reprinted: Virago, 1985).

1928. Some tribal origins, laws and customs of the Balkans, London: Allen and Unwin.

1990. Brenga e Balkanit dhe shkrime të tjera për Shqipërinë dhe Shqiptarët [The burden of the Balkans and other essays], Tirana: 8 Nëntori.

Elezi, Ismet. 1983. E D rejta zakonore penale e Shqiptarëve dhe lufta për zhdukjen e mbeturinave të saj në Shqipëri [L e droit coutumier pénal des Albanais]. Tirana: 8 Nëntori.

1994. E D rejta zakonore e L abërisë në planin krahasues [L e droit coutumier de $L$ abëria sur le plan comparatif]. Tirana: Libri Universitar.

Gjeçov, Shtjefën K. 1989. Kanuni i L ekë D ukagjinit [L e C ode de L eka D ukagjini], publié et annoté par K. Nova, Tirana: Académie des Sciences, Patrimoine Culturel des Albanais: Droit Coutumier, 1. (Orig. Shkodra, 1933. Trad. it.: Codice di Lek Dukagjini ossia diritto consuetidunario delle montagne d'Albania, Roma: Reale Academia, 1941, Centro di Studi per l'Albania, 2. Trad. all.: 'Das albanische Gewohnheitsrecht', Zeitschrift für vergleichende Rechtswissenschaft, 54-6 (1954-8). Trad. angl.: New York: Gjonlekaj Publishing Company, 1989).

Gurakuqi, Karl et Filip Fishta. 1937. Visaret e Kombit [L es trésors de la N ation]. Tirana.

Hahn, Johann Georg von. 1854. Albanesische Studien. Jena: Mauke.

Hasluck, Margaret M. Hardie. 1954. The unwritten law in Albania, ed. J. H. Hutton. Cambridge: Cambridge University Press.

Hecquard, Hyacinthe. 1858. H istoire et description de la H aute A lbanie ou G hégarie. Paris: Bertrand.

Lévi-Strauss, Claude. 1967. Les structures élémentaires de la parenté, Paris-La Haye: Mouton.

1983. Le regard éloigné. Paris: Plon.

Meillet, Antoine. 1920. M émoires de la Société de linguistique de Paris, 21: 45-8.

Nopcsa, Franzs. 1925. Albanien: Bauten, Trachten und G eräte N ordalbaniens, Berlin und Leipzig: de Gruyter.

1927. 'Ergänzungen zu meinem Buch über die Bauten, Trachten und Geräte Nordalbaniens'. Zeitschrift für Ethnologie 59: 279-81.

Poghirc, Cicerone. 1987. 'Albanian religion', in: The Encyclopedia of Religion, 1: 178-80.

Pulaha, Selami. 1975a. 'Kontribut për studimin e ngulitjes së katundeve dhe krijimin e fiseve në Shqipërinë e Veriut në shekujt 15-16' [Contribution à l'étude de l'établissement des villages et de la constitution des lignages en Albanie du Nord aux $15^{\mathrm{e}}$ et $16^{\mathrm{e}}$ siècles], Studime $\mathrm{H}$ istorike 1: $75-110$.

1975b. 'Mbi gjallërimin e lidhjeve farefisnore dhe krijimin et fiseve në Shqipërinë e Veriut në shekujt 16-17' [L'intensification des structures lignagères et la constitution des lignages en Albanie du Nord aux $16^{\mathrm{e}}$ et $17^{\mathrm{e}}$ siècles], Studime H istorike 2: 121-45.

1977. 'Formation des régions d'auto-gouvernement dans les Montagnes du Sandjak de Shkodra aux xve-xviie siècles', La Conférence $N$ ationale des Études Ethnographiques (28-30 juin 1976), 177-83. Tirana: Académie des Sciences.

Pupovci, Syrja. 1971. Marrëdhëniet juridike civile në Kanunin e Lekë Dukagjinit [Les relations juridiques civiles au Kanuni i L ekë D ukagjinit]. Prishtina: Enti TMM.

Seiner, Franz. 1922. Ergebnisse der Volkszählung in Albanien in dem von den österreichischungarischen Truppen 1916-1918 besetzten G ebiete. Wien.

Sicard, Emile 1943. La Zadruga sud-slave dans l'évolution du groupe domestique. Paris: Ophrys. (Thèse de Doctorat ès Lettres présentée à l'Université de Clermond-Ferrand.) 
Stahl, Paul Henri. 1977. 'Soi-même et les autres: quelques exemples balkaniques', in: L'I dentité. Séminaire dirigé par Claude Lévi-Strauss, 287-303. Paris: P.U.F.

1988. 'La regione tribale albanese', Incontri M eridionali 3:33-80.

Tirta, Mark. 1980. 'Aspects du culte des ancêtres et des morts chez les Albanais', Ethnographie Albanaise 10: 59-106.

Ulqini, Kahreman. 1977. 'Les rapports entre le bajraku, le fisi, et la région ethnographique', La conférence nationale des études ethnographiques (28-30 juin 1976), 617-19. Tirana: Académie des Sciences.

1985. 'L'activité de droit coutumier dans le cadre de l'institution de bajraku', Ethnographie Albanaise 14: 223-42.

1987. 'Survivances de l'ancienne organisation sociale à Himara et à Suli', Ethnographie albanaise 15: $197-222$.

1988. 'E drejta zakonore shqiptare përballe të drejtës kanonike dhe sheriatit' [Comparaison entre le droit coutumier albanais, la droit canonique et la sharia], Etnografia Shqiptare 16: 65-82.

Valentini, Giuseppe. 1956. II diritto delle comunità nella tradizione giuridica albanese, Firenze: Leo S. Olschi.

1965. La legge delle montagne albanesi nelle relazioni della missione volante, 1880-1932. Firenze: Leo S. Olschi.

Vroonen, Eugène. 1967. Les noms de famille dans le monde. Anthroponymie universelle comparée. Bruxelles: Librairie Encyclopédique.

Zojzi, Rrok. 1977. 'Survivances de l'ordre du fis-i dans quelques micro-régions de l'Albanie', in: A. Buda et al. (dir.), La conférence nationale des études ethnographiques (28- 30 juin 1976), 185-206. Tirana, Académie des Sciences.

Zonabend, Françoise. 1986. 'De la Famille. Regard ethnologique sur la parenté et la famille', in: $\mathrm{H}$ istoire de la famille (eds.) A. Burguière, C. Klapish-Zuber, M. Segalen et F. Zonabend. 1: 15-75. Paris: Armand Colin. 